\title{
Capacity to multitask limits cellular chemotaxis
}

Hye-ran Moon ${ }^{1, *}$, Soutick Saha ${ }^{2, *}$, Andrew Mugler ${ }^{2,3,4, a}$, and Bumsoo Han ${ }^{1,3,5, b}$

1 School of Mechanical Engineering, Purdue University, United States

2 Department of Physics and Astronomy, Purdue University, United States

3 Purdue Center for Cancer Research, Purdue University, United States

4 Department of Physics and Astronomy, University of Pittsburgh, United States

5 Weldon School of Biomedical Engineering, Purdue University, United States

*: These authors contributed equally.

Corresponding Authors: a : andrew.mugler@pitt.edu, b : bumsoo@purdue.edu, 


\begin{abstract}
Chemotaxis is the biased movement of cells in the direction of chemical gradients. Cellular signal processing capacity has been thought to be important to cells' chemotaxis behavior. However, it still remains elusive how cells sense and decipher multiple chemical cues. In this study, we test a hypothesis that the chemotaxis performance of cells is constrained by the capacity to "multitask." Specifically, if the intracellular signal processing capacity to respond to multiple cues is saturated, the effect of chemoattractants become antagonistic rather than synergistic or even independent. We experimentally investigate the migratory behavior of two types of cancer cells under single and combined cues of chemoattractants - transforming growth factor-beta and epidermal growth factor. For both cell types, the results show that the combination of the two attractants suppresses the chemotactic performance of cancer cells. We propose a novel biophysical framework that puts forward the hypothesis that the antagonism is not because of crosstalk, but because of the saturation of the intracellular signal processing capacity. The theory predicts that the suppression of chemotactic performance can alternatively be caused by saturating the signal processing capacity with background signal strength. We confirm this prediction with further experiments on both cell lines. Our framework provides new insight into the intracellular mechanism of signal processing and allows one to predict cellular response under complex chemical cues.
\end{abstract}




\section{Statement of Significance}

Cellular signal processing capacity is critical to understand cell chemotaxis behaviors — cell movement toward the direction of a chemical gradient signal. The signal environment exposing to cells is complex with multiple chemical cues. However, it is still elusive how cells sense and decipher multiple chemical signals. Here we experimentally investigate the chemotaxis performance of cells and propose a novel biophysical framework to reveal cells' signal processing capacity to "multitask". Is there a limit in the cellular multitasking capacity? We observe that overloaded environmental signal constraints chemotactic performance, specifically accuracy. Our results suggest that saturation of the signal processing capacity causes the suppression of the chemotactic accuracy. Our framework provides new insight into signal processing mechanisms to predict cellular response under complex chemical signals. 


\section{Introduction}

Cell chemotaxis - the biased migration of cells along a chemical cue - is a critical step in various pathological and physiological processes, including cancer metastasis, embryogenesis, and wound healing (1). For multiple cell types, including cancer, cells are exposed to multiple signals within complex chemical and physical environments during migration $(2,3)$. These include multiple cytokines such as transforming growth factor- $\beta$ (TGF- $\beta$ ), epidermal growth factor (EGF), stromal cell-derived factor-1 $\alpha(\mathrm{SDF}-1 \alpha)$, and CCL2 (2-5). The nature of the cytokine signals varies in spatial and temporal manner $(6,7)$. Cellular sensory function is critical to sense and decipher the complex signals to accurately migrate in the direction of physical and chemical cues. However, it is still not well understood how cells multitask while sensing complex signals to decide migratory directions.

The cellular function to sense chemical cues is achieved by intracellular signaling molecules and their downstream networks. Intracellular signaling networks involve a series of processes relevant to diverse cell physiological responses, including chemotaxis $(8,9)$. Research efforts to identify the associated signaling molecules and systematically construct their networks have advanced our understanding of how cells sense the signals resulting in chemotactic response $(2,6,10,11)$. For example, signal transduction in chemotaxis has been widely investigated with an amoeba model, Dictyostelium (11). G-protein mediated pathways activated by G-protein coupled receptors (GPCR) induce the localized accumulation of the signaling molecules such as phosphatidylinositol 3,4,5-trisphosphate $\left(\mathrm{PIP}_{3}\right)$, leading to Rac-mediated actin polymerization $(11,12)$. Similarly, receptor tyrosine kinases (RTK) -involved pathways such as Cofilin activation through EGFR in breast carcinoma cells mediate localized actin polymerization through signaling molecule 
accumulations $(9,12,13)$. The small GTPases within the Rho subfamily have been known to regulate actin polymerization, microtubule dynamics, and directional sensing, which play critical roles in chemotaxis $(11,14-16)$. Quantitative modeling involving chemical kinetics for the signaling molecules or logic models have extensively contributed to constructing signaling networks in systems biology (17). Furthermore, biophysical approaches have measured the cellular capacity in sensory function in a quantitative manner (18-20). Physical limits to signal sensing have been investigated considering the biochemical nature of signal-receptor binding relations (19, 20). Also, the effect of receptor crosstalk in enhancing the precision of chemical sensing of multiple ligands has been demonstrated with quantitative modeling (21). In cellular signal processing, intracellular protein diffusion and activation are critical to understanding the cellular sensory function (22). Not only the spatial variation, but temporal relations were used to develop a better understanding and physically predictable models (23). Despite their advances in elucidating cellular sensory function, these approaches are still limited in their ability to predict the chemotactic response to a complex signal environment.

In the cellular microenvironment, cells are exposed to multiple signals whose chemical composition and physical nature are complex. Indeed, cells sense and decipher multiple signals at the same time. Cellular multitasking capability has been addressed in functional crosstalk between either receptors or pathways (24-27). The EGFR cascade, which is an example of RTK, crosscommunicates with the GPCR-mediated chemotaxis pathways in breast cancer (24). Also, EGFR signaling can be induced by CXCR4 activation binding with stromal cell-derived factor-1 $\alpha$ (SDF$1 \alpha$, resulting in cooperative function in cancer metastasis (25). RTKs are also known to interplay with transforming growth factor- $\beta$ (TGF- $\beta$ ) through Smad-dependent transcription or PI3K/AKT activation (26). The crosstalk in either molecular or network levels are reflected in cellular 
responses. A cooperative effect of EGF and SDF-1 $\alpha$ gradients mediating breast cancer chemotaxis was reported by Mosadegh et al (28). In the study (28), breast cancer cells showed directional movement toward the SDF-1 $\alpha$ gradient when supplemented with EGF whereas migration of the cells was poorly stimulated by SDF-1 $\alpha$ gradient only. In addition, Uttamsingh et al (29) showed a synergistic effect of EGF and TGF- $\beta$ in inducing invasion/migration capability in epithelial cells. When the rat intestine epithelial cells were stimulated by both growth factors, the invasion/migration of cells in Boyden chamber was highly enhanced. The effect of TGF- $\beta$ inducing epithelial-mesenchymal transition was considered to cooperate with the CCL7-mediated chemotaxis (30). The combined effect of TGF- $\beta$, EGFR, and HER2 pathways was implemented to develop targeted therapy for pancreatic adenocarcinoma (31).

Despite the research advances in cellular signal processing, how cells multitask complex chemical signals is still poorly understood. Recent studies have shown crosstalk in signals and associated signaling pathways, implying a multitasking capability of cells in processing multiple signals (2427). However, the question still remains: Is there a limit to cellular multitasking capacity, and if so, what happens when cells exceed it?

In this study, we investigate how cells process and decipher multiple chemical cues. We hypothesize that cell multitasking capacity determines the cells' chemotactic accuracy under complex signals. To test this hypothesis, we experimentally assess the chemotaxis of cancer cells in response to multiple signals with various physical and chemical contexts. Migratory behaviors of breast cancer cells (MDA-MB-231) within a 3D matrix are assessed in response to single and multiple cues of TGF- $\beta 1$ (denoted as TGF- $\beta$ ) and EGF by use of a microfluidic platform. On the microfluidic platform, chemical cues of TGF- $\beta$ and EGF are created with different gradient 
strengths and background concentrations. Our observations are then validated by further experiments with a pancreatic cancer cell line. Based on the results, we develop a novel biophysical framework that can explain different types of chemotactic response in multiple-cue cell migration experiments and propose quantitatively testable predictions. The predictions are then confirmed with further experiments on both cell lines. 


\section{Materials and Methods}

\section{Cell and Reagents}

A human breast cancer cell line (MDA-MB-231) and a murine pancreatic cell line (eKIC) were used in this study. MDA-MB-231 cells were maintained in Dulbecco's Modified Eagle Medium/Ham's F-12 (Advanced DMEM/F-12, Lifetechnologies, CA, USA) supplemented with $5 \% \mathrm{v} / \mathrm{v}$ fetal bovine serum (FBS), $2 \mathrm{mM}$ L-glutamine (L-glu), and $100 \mu \mathrm{g} \mathrm{ml}^{-1}$ penicillin/streptomycin (P/S). A murine pancreatic cancer cell line, eKIC, was isolated from KIC mice, which were established as a genetically engineered mouse model of PDAC (GEM). For the KIC mice, Kras was combined with the deletion of the Ink4a locus (Ink4a/Arf $\left.f^{L / L}\right)$ to generate the Pdxl-Cre;LSL-Kras ${ }^{G 12 D} ;$ Ink4a/Arff- GEM $(32,33)$. The eKIC cells were cultured in RPMI 1640 with 2.05mM L-glutamine (GE Healthcare Bio-Sciences Corp., MA, USA) supplemented by 5\% $\mathrm{v} / \mathrm{v}$ fetal bovine serum (FBS) and $100 \mu \mathrm{g} \mathrm{ml}^{-1}$ penicillin/streptomycin (P/S). Both MDA-MB-231 and eKIC cells were regularly harvested by $0.05 \%$ trypsin and $0.53 \mathrm{mM}$ EDTA (Lifetechnologies, CA, USA) when grown to $\sim 80 \%$ confluency in $75 \mathrm{~cm}^{2} \mathrm{~T}$-flasks and incubated at $37^{\circ} \mathrm{C}$ with $5 \%$ $\mathrm{CO}_{2}$. The harvested cell suspensions were used for experiments or sub-cultured. Both cells were maintained below $15^{\text {th }}$ passage while regularly kept in cryopreservation.

\section{Chemotaxis assay}

For the chemotaxis assay, cells were implanted in the chemotaxis platform, which is an in vitro microfluidic device developed to engineer the chemical environment (Supporting Material). Cells were embedded uniformly to type I collagen mixture (Corning Inc., NY, USA) supplemented with 10X PBS, NaOH, HEPE solution, FBS, Glu, P/S, and cell-culture level distilled water. Initial cell density was consistently $5 \times 10^{5}$ cells $/ \mathrm{ml}$ for MDA-MB-231 and $8 \times 10^{5}$ cells $/ \mathrm{ml}$ for eKIC 
respectively in the $2 \mathrm{mg} / \mathrm{ml}$ type I collagen mixture. After loading, the cells in the collagen matrix were cultured with basic mediums for 24 hours. MDA-MB-231 cells were then exposed by serumreduced medium for another 24 hours. The serum-reduced medium was supplemented by $1 \% \mathrm{v} / \mathrm{v}$ FBS instead of 5\% v/v FBS in the basic medium. Due to a critical viability change in the serumreduced culture condition (data is not shown), serum starvation was not conducted for eKIC. Then, cells were exposed by concentration gradients of growth factors, either Transforming growth factor beta-1 (TGF- $\beta 1$, Invitrogen, CA, USA) or epidermal growth factor (EGF, Invitrogen, CA, USA).

\section{Characterization of cell migration}

Live-cell imaging technique with time-lapse microscopy was used to characterize cell migration. An inverted microscope (Olympus IX71, Japan) was equipped with a stage top incubator as described in (34), which allowed maintaining the microfluidic chemotaxis platform at $37^{\circ} \mathrm{C}$ with $5 \% \mathrm{CO}_{2}$ environment during imaging. MDA-MB-231 cells in the chemotaxis platform were captured every 15 minutes for 9 hours. eKIC cells were captured every 5 minutes for 3 hours. The temporal intervals and durations for the time-lapse imaging were optimized for each cell line by considering the cell motility, $\sim 12 \mu \mathrm{m} / \mathrm{h}$ for MDA-MB-231 and $\sim 50 \mu \mathrm{m} / \mathrm{h}$ for eKIC, respectively (Fig. S6 in the Supporting Material). In both cases, the time-lapse imaging was started 3 hours after applying the growth factors for sufficient adjusting time to develop gradient profiles accordingly (Supporting Material). The bright-field images were further processed to analyze cell migration. The cell area was defined by using the contrast differences between cells and background and converted to monochrome images by using ImageJ. A cell trajectory was illustrated as a collection of the centroid positions of cell areas at different time points. In tracking 
the cell movement, we excluded the cells undergoing division to avoid extra effect for cell polarity (35). Also, we excluded the stationary cells defined when the cells moved less than their diameter.

Biased cell motion is commonly characterized by directional accuracy, persistence, and motility (34). We measure directional accuracy using the chemotactic index (CI)

$$
C I=\cos \theta
$$

where $\theta$ is the angle between the net displacement of a trajectory and the gradient direction. The displacement is defined as a straight line connecting the initial and final points of a trajectory where we measure each trajectory for 9 hours (Fig. S1 B). The CI can range between -1 and 1 . Higher CI indicates more accurate chemotaxis in response to an attractant. $\mathrm{CI}=1$ means that the cell perfectly follows the gradient direction, whereas $\mathrm{CI}=0$ means that the cell is unbiased. Cells moving towards the opposite direction to the gradient have a negative CI between -1 and 0 . In an experimental trial, multiple CIs are distributed throughout the range of -1 to 1 due to variations of cell response to the attractant. While each CI represents the directional accuracy of a single trajectory, we measure a median $\mathrm{CI}$ as a representative $\mathrm{CI}$ in a trial. Our previous study has shown that the CI distribution is U-shaped without any attractant, indicating that the cell migration directions are uniformly distributed; in contrast, the CI distribution with an attractant shows a biased distribution toward 1 (34). In addition, motility is quantified as an instantaneous speed along the cell trajectory: 


$$
\text { Cell speed }=\frac{\text { Path } \text { of a trajectory }}{\text { Duration }}
$$

where the cell path is taken from a trajectory where measurement is taken every $\Delta t=15$ minutes. In our previous study for the physical constraints of the breast cancer chemotaxis (34), we have shown that the directional accuracy is a dominant metric in the chemotaxis of the $3 \mathrm{D}$ cultured cells migrating in ECM whereas cell persistence is barely changed by the chemical gradient. In this study, we mainly discuss the chemotaxis with the accuracy and motility, excluding the persistence.

\section{Statistical analysis}

In evaluating the chemotactic characteristics, more than 30 trajectories were analyzed in an experimental trial, which was repeated at least three times for all experimental groups. Each trajectory was quantified as CI and speed. To evaluate the chemotactic accuracy, medians of CIs from the repetitions were averaged and reported in a bar (Fig. 2B, 3A, and 5BC) with an error bar representing a standard estimated error (S.E.). For the simulation, CIs were collected from 400 cell trajectories in a control group and reported as an average \pm S.E. of the CIs of 400 trajectories. Differences in CIs presented in the bar graphs were statistically analyzed by student t-test. The significant changes between comparisons were examined when the $p$ value $<0.05$. In addition, all collected CIs and speeds in a group were reported in box plots. For the speeds in Fig.2C, 3B, and Fig.S6, Tukey's post hoc test in ANOVA was used to assess the statistical significance when the $\mathrm{p}$ value $<0.05$. A data point in the box plots in Fig. $\mathbf{S 5}$ and Fig. S6 indicates each metric of a cell trajectory showing the distribution characteristics of the metric in a group. 


\section{Results}

Multitasking of breast cancer cells results in antagonism under combined TGF- $\beta$ and EGF gradients

Gradients of diverse soluble factors, including TGF- $\beta$ and EGF, guide biased cell motion in vivo $(2,6,7)$. The gradient sensing results in the biased movement of the cells through associated signal pathways. In the signal processing, complex signal transductions converge to functional molecules such as $\mathrm{PIP}_{3}$ in Dictyostelium and cofilin in breast carcinoma cells (11). Conceptually, representing a functional molecule as $\mathrm{M}$, the chemotactic response consists of a signal-receptor binding, which activates $\mathrm{M}$ to $\mathrm{M}^{*}$, ultimately leading to chemotaxis, as illustrated in Fig. 1. To understand cellular multitasking capability in regulating the cell chemotactic response to multiple signals, we consider various signal environments. Here, we hypothesize that cell chemotaxis is regulated not only by the biochemical nature of signals, but also by physical characteristics, including signal strength and background levels of the chemical cues. The multitasking capacity of cells is anticipated to either synergistically or antagonistically affect the cell chemotactic performance. 

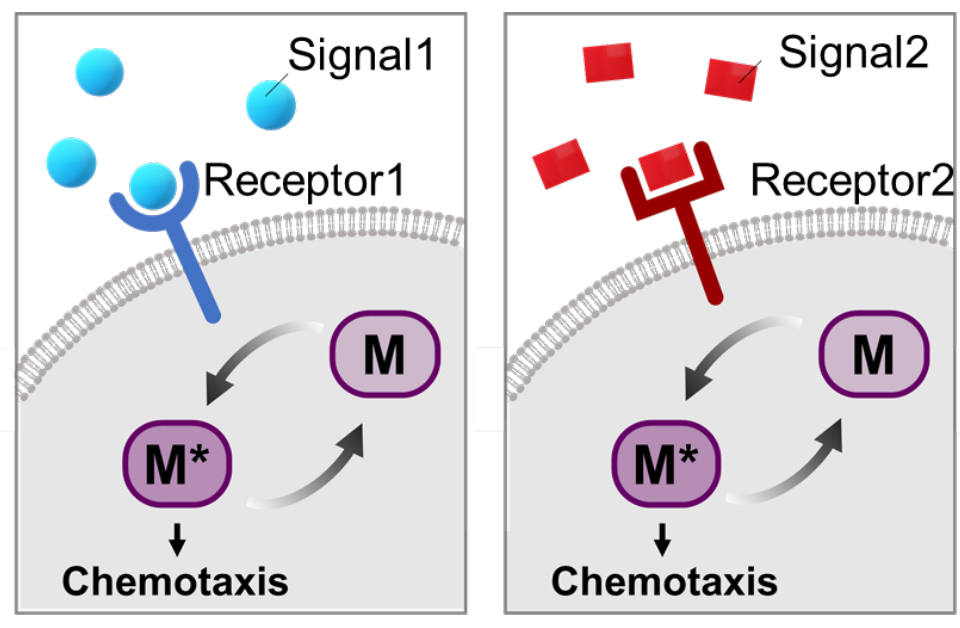

\section{Cellular multitasking}

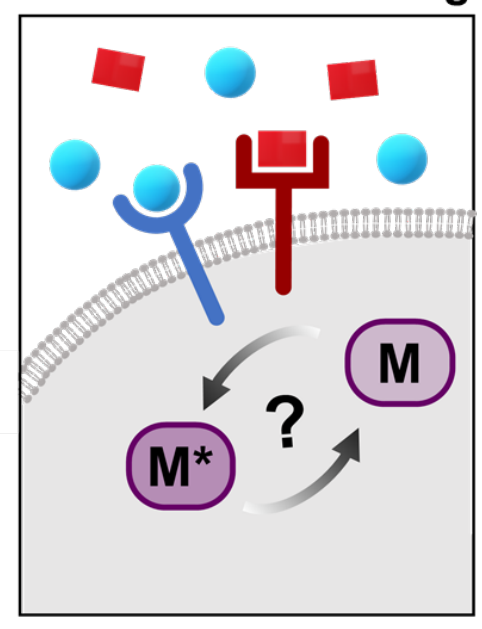

Fig. 1. Schematic of cellular multitasking for chemotaxis. Cells process each individual signal through functional molecules $\left(\mathrm{M}^{*}\right.$ activated from $\mathrm{M}$ through signal-receptor bindings). Cellular multitasking indicates cellular processing function for simultaneously exposed multiple signals. 
To investigate the multitasking capacity of the cells in response to the complex environmental signals, we present multiple signals simultaneously. For that, we use a triple-negative breast cancer cell line MDA-MB-231, which has been recognized as a highly metastatic cell type (36). Chemotactic response of MDA-MB-231 has been reported with several growth factors, including TGF- $\beta$ and EGF $(34,37)$. To elucidate the effect of combined signals on chemotaxis, we expose both TGF- $\beta$ and EGF gradients simultaneously to the cells using the chemotaxis platform (Supporting Material), track their migration (Fig. 2A), and compute the chemotactic index (the cosine of the angle between their displacement and the gradient direction). TGF- $\beta$ and EGF bind to independent receptors on the MDA-MB-231 cell surface. $(8,9)$ TGF- $\beta$ binds to the TGF- $\beta$ receptors, which are composed of type 1 and type 2 subunits. The TGF- $\beta$ binding receptors are transmembrane serine/threonine kinase complex (8). On the other hand, EGF binds EGFR which is a receptor tyrosine kinase superfamily (9). Surprisingly, the chemotactic index (CI) in response to the combined gradients decreases compared the response to each individual gradient (Fig. 2B), which suggests that multitasking can restrain the chemotactic performance, specifically the cell directional accuracy. On the other hand, cells under the combined gradients still show significantly enhanced speed compared to control, as do cells in the individual gradient cases (Fig. 2C). It is a remarkable result since many studies have reported the functional cooperation of multiple signals rather than the antagonism that we see here $(29,30)$. It is possible that although breast cancer cells do not share the same receptors for sensing TGF- $\beta$ and EGF gradients, there might be a convergence downstream in the signaling pathway that governs biased migration, common to both cues. 
A

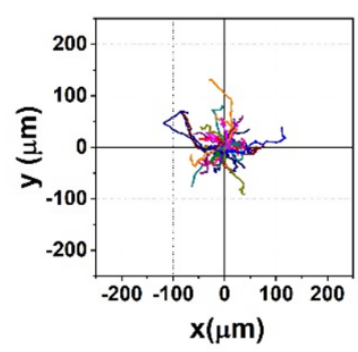

B

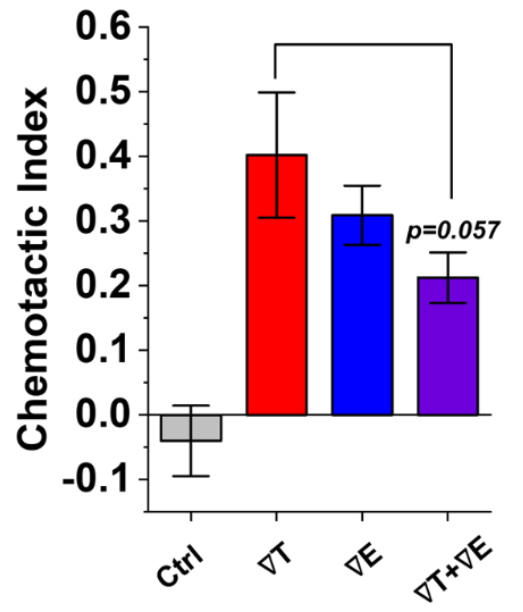

TGF $-\beta$ gradient
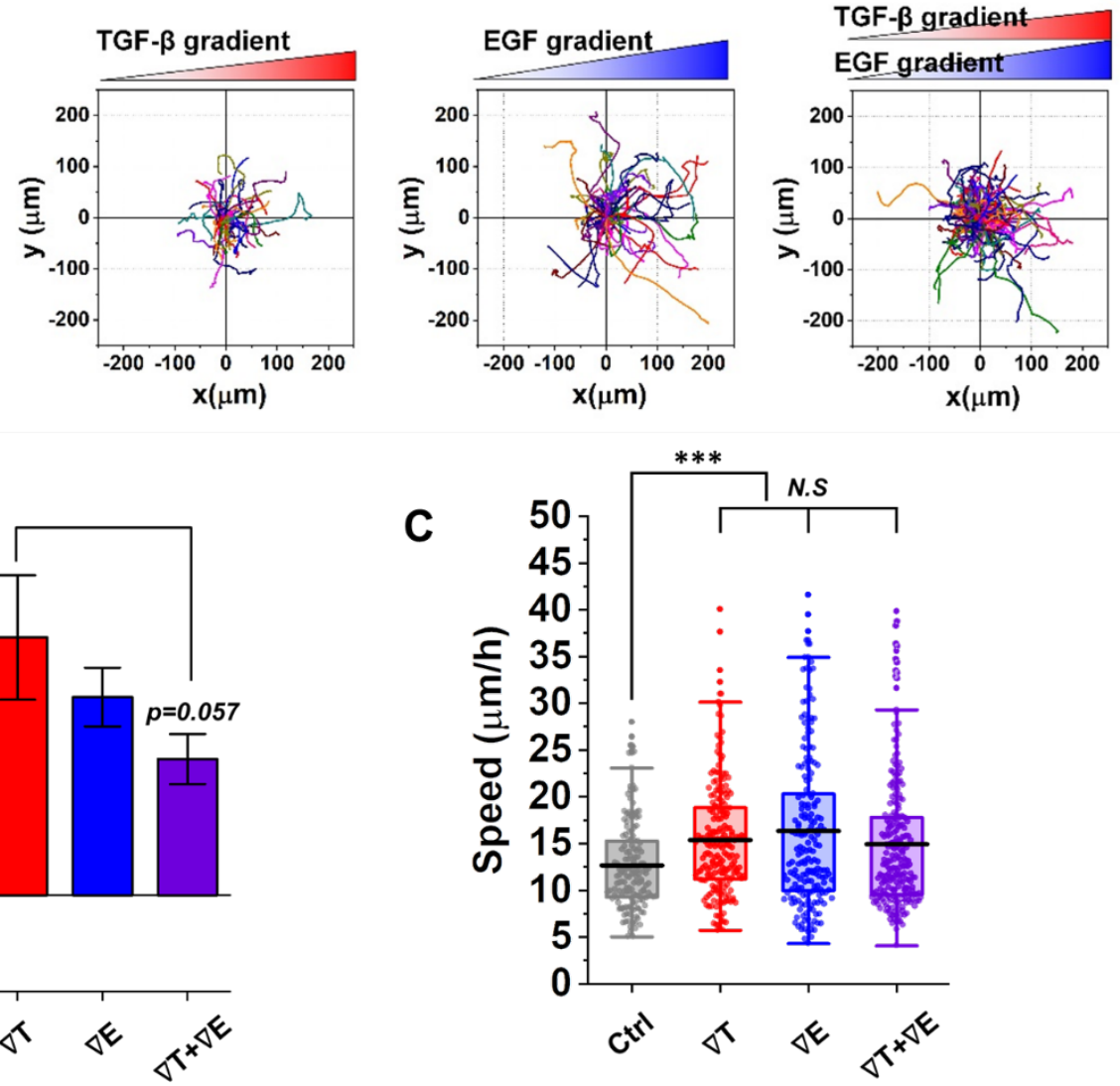

Fig. 2. Breast cancer cells chemotax up individual TGF- $\beta$ and EGF gradients more strongly than when gradients are combined (antagonism). (A) Cell trajectories of a representative sample for control, $50 \mathrm{nM} / \mathrm{mm}$ TGF- $\beta$ gradient $(\nabla T), 800 \mathrm{nM} / \mathrm{mm}$ EGF gradient ( $\nabla E)$, and combined gradients of $50 \mathrm{nM} / \mathrm{mm}$ TGF- $\beta$ with $800 \mathrm{nM} / \mathrm{mm}$ EGF $(\nabla T+\nabla E)$ collected during 9 hours Trajectories in a sample includes $>35$ cells. (B) Averages of medians of chemotactic index (Cl). Median indicates representative $\mathrm{Cl}$ of trajectories collected from an experimental sample (>35 trajectories/sample). Bar data represents mean \pm S.E $(n \geq 3$ independent $s$ amples / condition). Difference between Cls of $\nabla T$ and $\nabla T+\nabla E$ show close to statistical significance with $p=0.057$ in student t-test. (C) Speed distribution of all trajectories from 158-233 cells /condition. Dot represents speed from a single trajectory. Box represents quartiles with a mean line in the middle of the box. $\left(^{* * *}: p<0.001\right.$, N.S: no significance with $p>0.05$ in Tukey's post hoc test in ANOVA) 


\section{The limit to cellular multitasking capacity is not cell-type specific}

To evaluate the cell-type dependency in cellular multitasking, we investigate multiple-cue chemotaxis in pancreatic cancer cells. eKIC is used in this study, which is a murine pancreatic cancer cell line. eKIC is driven by the genetically engineered mouse model having Kras and p16 mutations showing epithelial phenotype $(38,39)$. The cell line is known to be responsive to TGF$\beta$ sensitively, showing invasion features (39). First, we evaluate the migratory behaviors of eKIC in the chemotaxis platform as a control, excluding any growth factors. The trajectories show an average CI close to 0, as shown in Fig. 3A (gray).

Then, we expose a single cue of the TGF- $\beta$ gradient and EGF gradient. We find that the eKICs are highly responsive to both TGF- $\beta$ gradient and EGF gradient (Fig. 3A, red and blue). Indeed, the CI values are significantly enhanced in both gradient groups compared to the control group. Interestingly, we observe the antagonism in eKIC in the presence of the combined TGF- $\beta$ and EGF gradients: the CI is significantly lower than the CIs of both groups of a single gradient (Fig. 3A, purple). The speed is similarly enhanced over the control (Fig. 3B). The antagonism indicates that the disruption of chemotactic performance is not a cell-type-specific response. The present results suggest that the cell's multitasking capacity might be physically governed by the limitation of a convergent downstream signaling process, resulting in disruption of chemotactic performance in both breast cancer and pancreatic cancer cells. 
A

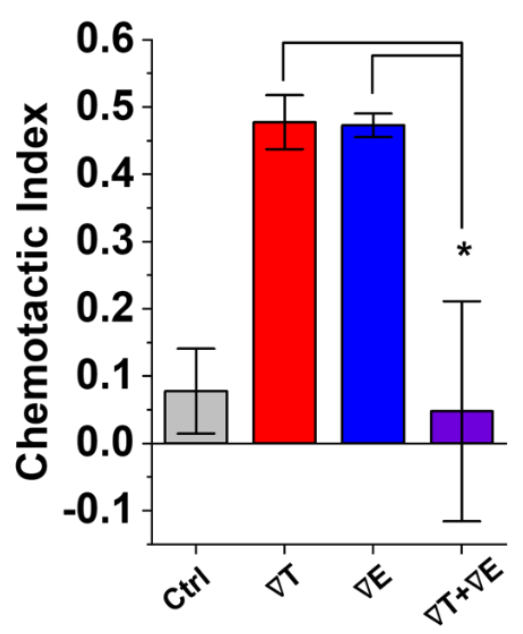

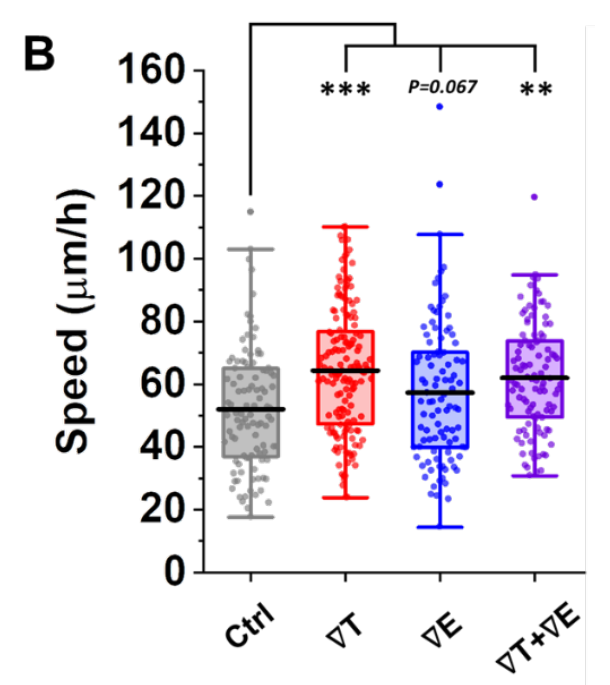

Fig. 3 Antagonism of overloaded signal environment in pancreatic cancer cells. (A) Averages of medians of chemotaxis index $(\mathrm{Cl})$ in control, $10 \mathrm{nM} / \mathrm{mm}$ TGF- $\beta$ gradient $(\nabla T)$, $200 \mathrm{nM} / \mathrm{mm}$ EGF gradient ( $\nabla E)$, combined gradients of $10 \mathrm{nM} / \mathrm{mm}$ TGF- $\beta$ and $200 \mathrm{nM} / \mathrm{mm}$ EGF $(\nabla T+\nabla E)$, collected during 3 hours. Bar represents mean of $\pm S . E(n \geq 3)$. The medians are collected from $>30$ trajectories in a sample, respectively. Difference between $\mathrm{Cls}$ shows statistical significance in student t-test $\left({ }^{*}: p<.05\right)$. (B) Speed distribution of all trajectories collected from $>100$ cells /condition; Dot: speed from a single trajectory. Box: interquartile range (IQR) \pm 1.5 IQR whiskers with a mean line in the middle of the box. ${ }^{*}: p<.05,{ }^{* *}:<.01,{ }^{* * *}: p<.001$ (ANOVA Tukey's post hoc test). 


\section{Cellular multitasking capacity can be explained by the presence of a common saturating component}

To understand the experimental results, we propose a simple model to describe biased cell migration in response to multiple chemical gradients. The most natural hypothesis for a reaction pathway that can lead to the observed antagonism under both cues is mutual repression, an example of crosstalk: TGF- $\beta$ would activate migration through pathway one, and EGF through pathway two, but TGF- $\beta$ would also repress pathway two and vice versa. However, we lack explicit experimental evidence of crosstalk in these cell lines. Moreover, the observation that the antagonism is not cell-type-specific suggests that we seek an explanation that is not sensitive to the particular details of the signaling network. Therefore, we propose a simpler mechanism that is based on the saturation of a common pathway component. We suppose that there is a molecular species M common to both the EGF and TGF- $\beta$ pathways that drives the cell migratory response. $M$ is converted to an active form $M^{*}$ by either EGF (E) or TGF- $\beta(T)$, and $M^{*}$ then propagates the signal further downstream in the pathway (Fig. 4A, left). In our model, $\mathrm{M}$ is converted to $\mathrm{M}^{*}$ with rate $k_{\mathrm{T}}$ by TGF- $\beta$ and with rate $k_{\mathrm{E}}$ by EGF, and $\mathrm{M}^{*}$ is converted to $\mathrm{M}$ with a rate of 1 which sets the timescale for the system:

$$
T+M \stackrel{k_{T}}{\rightarrow} T+M^{*} ; \quad E+M \stackrel{k_{E}}{\rightarrow} E+M^{*} ; \quad M^{*} \stackrel{1}{\rightarrow} M
$$

A TGF- $\beta$ or EGF gradient is translated into a difference in $M^{*}$ molecules between the forward and backward halves of the cell (Fig. 4A, right). We therefore take the difference $\Delta \mathrm{M}^{*}$ to be the output of the model. In our model we do not explicitly take intracellular diffusion of the $\mathrm{M}^{*}$ molecule into account. But in choosing the difference of $\mathrm{M}^{*}$ molecules between the two halves as the output signal of interest we are implicitly assuming fast diffusion of an internal messenger within the cell which makes the subtraction of $\mathrm{M}^{*}$ molecules in the two halves possible $(40,41)$. We also assume 
that the receptors on the cell diffuse much slower compared to any other relevant timescale in our model to make the sensing of the external chemical gradient possible. The statistics of $\Delta \mathrm{M}^{*}$ can be found by recognizing that conversion of $\mathrm{M}$ to $\mathrm{M}^{*}$ or vice versa is a binomial process (Supporting Material). In steady state, the signal to noise ratio (SNR) is given by,

$$
S N R=\frac{\overline{\Delta M^{*}}}{\sigma_{\Delta M^{*}}}=\frac{\epsilon}{1+\eta+\beta} \sqrt{\frac{M_{0} \beta(1+\eta)}{2}}
$$

where the dimensionless parameters $\eta, \beta$, and $\epsilon$ are defined as,

$$
\eta=\frac{k_{T} T_{0}}{k_{E} E_{0}} ; \quad \beta=\frac{1}{k_{E} V E_{0}} ; \quad \epsilon=\frac{R g_{T}}{T_{0}}=\frac{R g_{E}}{E_{0}}
$$

Here $T_{0}$ and $E_{0}$ are the background concentrations of TGF- $\beta$ and EGF at the center of the cell, respectively; $g_{\mathrm{T}}$ and $g_{\mathrm{E}}$ are the gradients of TGF- $\beta$ and $\mathrm{EGF}$, respectively; $V=4 \pi R^{3} / 3$ is the volume of the spherical cell; and $M_{0}$ is the total number of $\mathrm{M}$ and $\mathrm{M}^{*}$ molecules in the cell, which is assumed to be constant. Using $\epsilon$ directly from experiments, and calibrating $\eta$ to produce a stronger response with a TGF- $\beta$ gradient alone than with an EGF gradient alone as in the experiments, we find that there is a range of $\beta$ values for which this simple model exhibits antagonism for the SNR. An example is shown in Fig. 4B, and more generally, antagonism occurs for $\beta<[\eta(1+\eta)]^{1 / 2}$ if $\eta<$ 1 and for $\beta<(1+\eta)^{1 / 2}$ if $\eta \geq 1$. The intuitive reason that antagonism occurs at small $\beta$ is that small $\beta$ corresponds to strong conversion rates of $\mathrm{M}$ to $\mathrm{M}^{*}$. This means that most molecules are in the $M^{*}$ state, i.e., the pathway is close to saturated. With only one gradient signal, the pathway is not yet fully saturated, and there are more $\mathrm{M}^{*}$ molecules produced in the front of the cell than in the 
back. However, with both signals, the pathway becomes saturated, and the number of $\mathrm{M}^{*}$ molecules is close to its maximal value in both halves, leading to a reduced difference $\Delta \mathrm{M}^{*}$.

The mathematical model gives the SNR, and while it is reasonable to assume that CI is a monotonically increasing function of the SNR (42), we would like to make predictions for the CI and its associated statistics directly. To do so, we develop a simulation of the cell response based on the mathematical model. We explicitly simulate the reactions in Eq. 3, where $\mathrm{T}$ and $\mathrm{E}$ are modeled as continuous concentrations, and $\mathrm{M}$ and $\mathrm{M}^{*}$ are modeled as a fixed number $M_{0}$ of discrete molecules uniformly distributed around the surface of a circular cell, as illustrated in Fig. 4A (right) (We show in the Supporting Material that results for a circular cell executing a 2D biased random walk are similar to a spherical cell executing a 3D biased random walk). The direction of movement $\theta$ is chosen according to the distribution of $\mathrm{M}^{*}$ on the surface, and the cell moves at a constant speed until the next reaction takes place. We calculate the CI from the simulated trajectory in the same way it is calculated in the experiments. Using the same parameters $\epsilon, \eta$, and $\beta$ as in Fig. $\mathbf{4 B}$, and fixing $M_{0}$ to calibrate the CI to the TGF- $\beta$ case in the experiments (Fig. S3), we find that our simulation results exhibit antagonism, consistent with the experiments (Fig. 4C). The full distributions of CI from the simulations are shown in Fig. 4D and show agreement with those from the experiments (Fig. S5). 
A

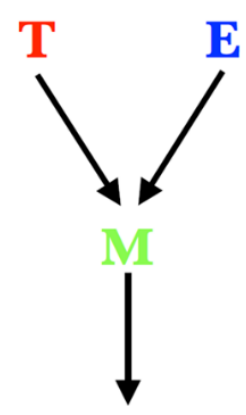

migration

M*

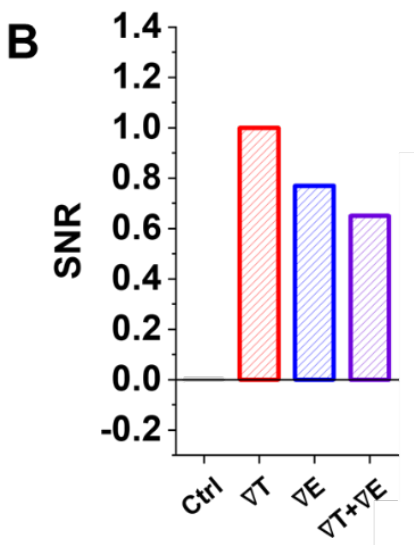

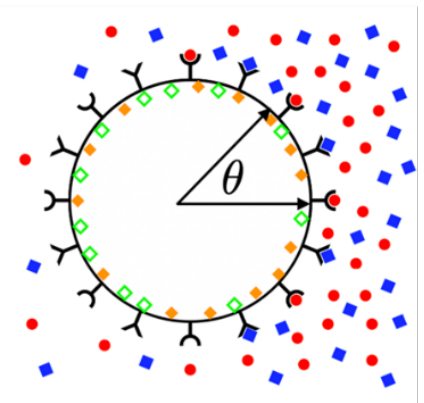

- $T \diamond M$

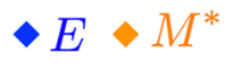

C

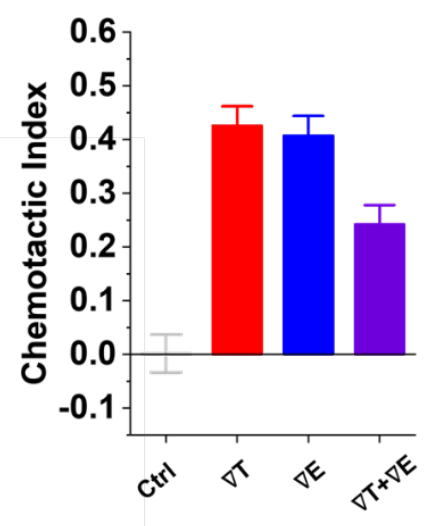

Fig. 4. Mathematical model explains antagonism by saturation of common pathway component. (A) Schematic of model. T, TGF- $\beta$; E, EGF, M, common component; $\mathrm{M}^{*}$, activated form. (B) Signal to noise ratio (SNR) is lower for combined gradients than individual gradients (antagonism). Parameter values: $\eta=0.5, \beta=0.1$. SNR is with respect to the signal to noise ratio when only TGF- $\beta$ gradient is present. (C) Simulation of model reactions reproduces experimentally observed antagonism in chemotactic index $(\mathrm{Cl})$ at level of medians. Bar: medians \pm S.E 


\section{The biophysical framework successfully predicts cellular chemotactic responses to an "overloaded" signal environment}

To evaluate the hypothesis that saturation of a common pathway component causes the antagonism, we develop two experimentally testable predictions. Both predictions are based on the fact that saturation presents a tradeoff: the benefit of adding an EGF gradient to the TGF- $\beta$ gradient is that it presents a stronger directional cue; the cost is that it "overloads" the signaling network by pushing the number of activated molecules on each side of the cell closer to its maximal value and therefore taking the difference closer to zero. The cost wins, causing the antagonism. This intuition implies that if a uniform EGF signal is used instead of the EGF gradient, then the benefit is removed, and the CI should be further reduced. Indeed, the simulation results in Fig. $\mathbf{5 A}$ (black) show that the CI is significantly suppressed when adding a uniform EGF background concentration to the TGF- $\beta$ gradient. The previous result does not rule out the possibility that the EGF pathway specifically represses the TGF- $\beta$ pathway to create the antagonism. To disambiguate the saturation mechanism from the possibility of repressive crosstalk, we consider overloading the cell by adding to the TGF- $\beta$ gradient a uniform background concentration of TGF- $\beta$ instead of EGF. The intuition behind the saturation mechanism still implies that the CI should decrease, and indeed the simulation results in Fig. 5A (brown) show that the CI is significantly suppressed when adding a uniform TGF- $\beta$ background concentration to the TGF- $\beta$ gradient.

To validate the predictions, we investigate the effect of a background level of chemical signals experimentally. First, MDA-MB- 231 cells show that the CI is significantly reduced when the cells are exposed to $50 \mathrm{nM} / \mathrm{mm}$ of TGF- $\beta$ gradient in the presence of a uniform $400 \mathrm{nM}$ EGF background concentration (Fig. 5B, black). eKIC cells exposed to TGF- $\beta$ gradient $(10 \mathrm{nM} / \mathrm{mm})$ in the presence of a uniform EGF background concentration (100nM) also show significantly suppressed CI (Fig. 
5C, black). Because TGF- $\beta$ and EGF bind to different receptors, this result implies that neither receptor is being saturated. Instead, it validates our hypothesis that cellular multitasking is ineffective for the overloaded signal environment due to a limit in the convergent downstream of signal processing. Then, each cell type is exposed to TGF- $\beta$ gradient in the presence of a uniform TGF- $\beta$ background concentration. The results show that the CI is significantly reduced for both MDA-MB-231 and eKIC, as shown in Fig. 5B and C (brown), supporting the hypothesis that the antagonism is not due to repressive crosstalk but rather due to the saturation of a common pathway component. For both cases, cell speed is significantly enhanced compared to control (Fig. S6 Supporting Material). The present results suggest that cellular multitasking is limited in terms of chemotactic accuracy under the higher background signals. In contrast, the cell speed can still be enhanced in the overloaded signal environment.

In summary, the model successfully predicts the cellular response regardless of cell types. The chemotaxis is critically moderated by not only the environmental cues but also the intrinsic cell capability to process information within an inherently limited channel. Specifically, genetic mutations commonly observed in the cancer cells are highly relevant to exhibit the invasion process with specific signaling pathways such as Snail and Twist (43). Yet, the present results show that the cell response could be illustrated with the predictions from our framework regardless of the cancer type, implying the cellular multitasking capacity is not a cancer type-specific signaling effect. 

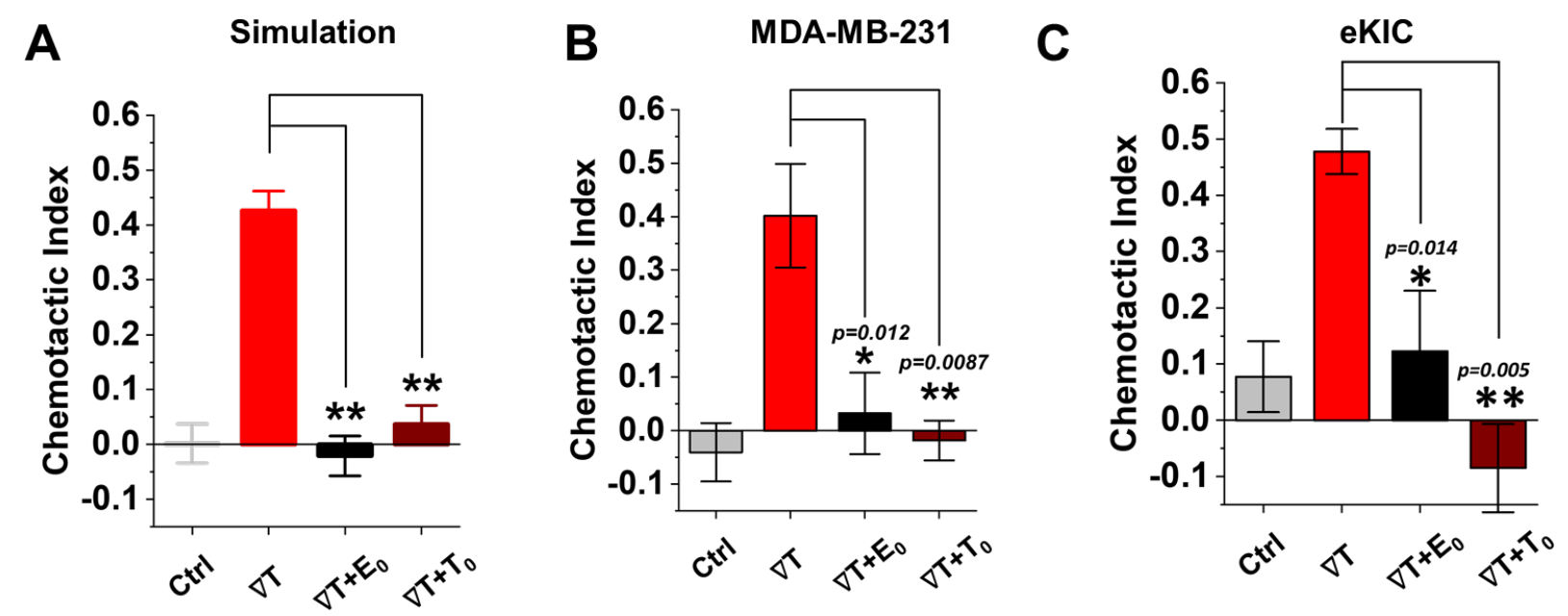

Fig. 5. Model prediction for an effect of signal background on the chemotaxis up a TGF- $\beta$ gradient and experimental validation. Chemotactic index $(\mathrm{Cl})$ from $(A)$ simulation and $(B, C)$ experiment show that chemotaxis up TGF- $\beta$ gradient is highly suppressed with the addition of a uniform background concentration regardless the signal type $\left({ }^{*}: p<.05,{ }^{* *}: p<.01\right.$ in Student t-test). In experiment, (B) MDA-MB-231 and (C) eKIC. Bar represents mean of the medians $\pm S . E(n \geq 3)$. The medians are collected from $>35$ trajectories in a sample, respectively. Dot represents $\mathrm{Cl}$ from a single trajectory. 


\section{Discussion}

The present results elucidate cellular multitasking capacity in an overloaded signal environment: the combined TGF- $\beta$ and EGF gradients, TGF- $\beta$ gradient with the elevated EGF background level, and TGF- $\beta$ gradient with the elevated TGF- $\beta$ background level. The chemotactic accuracy of the breast and pancreatic cancer cells showed significant suppression in the overloaded signal environment. To understand the cellular multitasking capacity, we proposed the biophysical framework with a simple mathematical model and simulation by suggesting the saturation of a common pathway component. In the framework, each growth factor competes for activation of a motility factor responsible for cell migration $\mathrm{M}^{*}$. The simple competitive binding model enables several surprising predictions. First, the simultaneously exposed TGF- $\beta$ and EGF gradients can induce chemotaxis with a weaker accuracy than the individual responses, addressing the antagonism found in the experiment. Also, the higher background noise causing the saturation of the motility factor elicits the loss of chemotactic accuracy in the cell response. The predictions were well validated by the experiment with strikingly similar results, suggesting the capability of the new framework to encompass many types of chemotaxis.

The present results elicit the antagonism of the combined cues, TGF- $\beta$ and EGF gradients, in chemotactic accuracy of cancer cells. The chemotactic accuracy of the cancer cells showed significant suppression when the TGF- $\beta$ and EGF gradients were simultaneously exposed to the breast and pancreatic cancer cells in the in vitro chemotaxis platform. Both growth factors have been identified to regulate cell invasiveness and motility $(2,44,45)$. In particular, the TGF- $\beta$ has been well recognized to promote epithelial-mesenchymal transition, which is a critical step in cancer invasion $(8,44)$. The role of TGF- $\beta$ in stimulating cancer progression and invasion has been considered as a cooperative effector when it combines with the other growth factors. $(29,30)$. 
Uttamsingh. et al., (29) reported that the roles of TGF- $\beta$ and EGF-mediated signaling pathways incorporate synergistically in inducing cell invasion and migration. However, the synergistic effect of TGF- $\beta$ with EGF is a result of the intrinsic capability relevant to cell motility, not fully addressing the cell chemotactic response. On the other hand, our results showed the effect of combined cues specifically for the cell chemotaxis as a systematic response. Indeed, cell chemotaxis is regulated by not only the intrinsic motility but also the physical aspects $(34,46,47)$. We observed the antagonism of TGF- $\beta$ and EGF gradients mainly in the cell chemotactic accuracy, while not in the motility. Moreover, the antagonism was shown in the highly noisy environment in detecting the chemical cues, indicating the biophysical aspect of the chemotaxis. Therefore, the antagonism in this study is a novel discovery, suggesting the necessity of a quantitative approach to multiple cue migration studies.

The biophysical framework demonstrates the combined action of multiple cues simply by focusing on the functional molecules which directly regulate cell chemotaxis. The chemotaxis is a systematic activity of intrinsic cellular responses, including actin polymerization and organization, cytoskeleton dynamics, and adhesion $(2,7,11)$. Recent advances have reported several signaling pathways regulating chemotaxis $(14,15,48,49)$. The localization of the relevant proteins in either the leading edge or the rear regulates the cell polarity and motility. Such proteins are activated when the environmental signals are detected and regulate the F-actin polymerization, myosin, microtubules, and adhesion. The complex process is illustrated simply in our model by using the common pathway components $\mathrm{M}$ and $\mathrm{M}^{*}$. In this matter, our framework provides new insights to elucidate the cellular process involved in chemotaxis in a simple and quantitatively testable way. 
shown in the experiment, further research is warranted. The present framework may limit the explanation regarding a mode of migration. The physical constraint driven by ECM regulates the migrating features as well. Since the chemotactic response and relevant signaling pathway inducing the chemotaxis could be varied depending on the mode of the migration $(47,48)$, the model may be improved further by considering the modes highly regulated by the physical confinement. Some mechanistic study may also improve the concept in integrating the biophysical framework with the signaling networks for chemotaxis by finding the potential molecules corresponding with $\mathrm{M}$ and $\mathrm{M}^{*}$. We presume that the functional molecules which regulate the chemotactic movement including the actin-cytoskeleton, microtubules, cell adhesion dynamics could be the potential molecules of $\mathrm{M}$ and $\mathrm{M}^{*}$, possibly found in downstream networks of Rho GTPases, Smad-dependent transcription, or PI3K/AKT activation cascades $(10,11,14-16,24-26$, $50)$. 


\section{References}

1. Welch, D. R., and D. R. Hurst. 2019. Defining the hallmarks of metastasis. Cancer research 79(12):3011-3027.

2. Roussos, E. T., J. S. Condeelis, and A. Patsialou. 2011. Chemotaxis in cancer. Nature Reviews Cancer 11(8):573-587.

3. Quail, D. F., and J. A. Joyce. 2013. Microenvironmental regulation of tumor progression and metastasis. Nature Medicine 19(11):1423-1437.

4. Mantovani, A., S. Sozzani, M. Locati, P. Allavena, and A. Sica. 2002. Macrophage polarization: tumor-associated macrophages as a paradigm for polarized M2 mononuclear phagocytes. Trends in immunology 23(11):549-555.

5. Fernandis, A. Z., A. Prasad, H. Band, R. Klösel, and R. K. Ganju. 2004. Regulation of CXCR4-mediated chemotaxis and chemoinvasion of breast cancer cells. Oncogene 23(1):157-167.

6. Odenthal, J., R. Takes, and P. Friedl. 2016. Plasticity of tumor cell invasion: governance by growth factors and cytokines. Carcinogenesis 37(12):1117-1128.

7. Van Haastert, P. J. M., and P. N. Devreotes. 2004. Chemotaxis: signalling the way forward. Nature Reviews Molecular Cell Biology 5(8):626-634.

8. Ikushima, H., and K. Miyazono. 2010. TGF $\beta$ signalling: a complex web in cancer progression. Nature Reviews Cancer 10(6):415-424.

9. Han, W., and H.-W. Lo. 2012. Landscape of EGFR signaling network in human cancers: biology and therapeutic response in relation to receptor subcellular locations. Cancer letters 318(2):124-134.

10. Miyagawa, T., H. Koteishi, Y. Kamimura, Y. Miyanaga, K. Takeshita, A. Nakagawa, and M. Ueda. 2018. Structural basis of Gip1 for cytosolic sequestration of G protein in widerange chemotaxis. Nature communications 9(1):1-13.

11. Swaney, K. F., C.-H. Huang, and P. N. Devreotes. 2010. Eukaryotic Chemotaxis: A Network of Signaling Pathways Controls Motility, Directional Sensing, and Polarity. Annual Review of Biophysics 39(1):265-289.

12. Kölsch, V., P. G. Charest, and R. A. Firtel. 2008. The regulation of cell motility and chemotaxis by phospholipid signaling. Journal of cell science 121(5):551-559.

13. Wang, W., R. Eddy, and J. Condeelis. 2007. The cofilin pathway in breast cancer invasion and metastasis. Nature Reviews Cancer 7(6):429-440.

14. Charest, P. G., and R. A. Firtel. 2007. Big roles for small GTPases in the control of directed cell movement. Biochemical Journal 401(2):377-390.

15. Chung, C. Y., S. Funamoto, and R. A. Firtel. 2001. Signaling pathways controlling cell polarity and chemotaxis. Trends in biochemical sciences 26(9):557-566.

16. Li, H., L. Yang, H. Fu, J. Yan, Y. Wang, H. Guo, X. Hao, X. Xu, T. Jin, and N. Zhang. 2013. Association between Gai2 and ELMO1/Dock180 connects chemokine signalling 
with Rac activation and metastasis. Nature communications 4(1):1-12.

17. Le Novère, N. 2015. Quantitative and logic modelling of molecular and gene networks. Nature Reviews Genetics 16(3):146-158.

18. Thomas, P. J., and A. W. Eckford. 2016. Capacity of a simple intercellular signal transduction channel. IEEE Transactions on information Theory 62(12):7358-7382.

19. Hu, B., W. Chen, W.-J. Rappel, and H. Levine. 2010. Physical limits on cellular sensing of spatial gradients. Physical review letters 105(4):048104.

20. Varennes, J., and A. Mugler. 2016. Sense and sensitivity: physical limits to multicellular sensing, migration, and drug response. Molecular pharmaceutics 13(7):2224-2232.

21. Carballo-Pacheco, M., J. Desponds, T. Gavrilchenko, A. Mayer, R. Prizak, G. Reddy, I. Nemenman, and T. Mora. 2019. Receptor crosstalk improves concentration sensing of multiple ligands. Physical Review E 99(2):022423.

22. Soh, S., M. Byrska, K. Kandere-Grzybowska, and B. A. Grzybowski. 2010. Reactiondiffusion systems in intracellular molecular transport and control. Angewandte Chemie International Edition 49(25):4170-4198.

23. Gupta, S., S. Fancher, H. C. Korswagen, and A. Mugler. 2020. Temporal precision of molecular events with regulation and feedback. Physical Review E 101(6):062420.

24. Hart, S., O. M. Fischer, N. Prenzel, E. Zwick-Wallasch, M. Schneider, L. Hennighausen, and A. Ullrich. 2005. GPCR-induced migration of breast carcinoma cells depends on both EGFR signal transactivation and EGFR-independent pathways. Biological chemistry 386(9):845-855.

25. Lappano, R., and M. Maggiolini. 2011. G protein-coupled receptors: novel targets for drug discovery in cancer. Nature reviews Drug discovery 10(1):47-60.

26. Shi, Q., and Y.-G. Chen. 2017. Interplay between TGF- $\beta$ signaling and receptor tyrosine kinases in tumor development. Science China Life Sciences 60(10):1133-1141.

27. Wang, Y., C. L. Chen, and M. Iijima. 2011. Signaling mechanisms for chemotaxis. Development, growth \& differentiation 53(4):495-502.

28. Mosadegh, B., W. Saadi, S. J. Wang, and N. L. Jeon. 2008. Epidermal growth factor promotes breast cancer cell chemotaxis in CXCL12 gradients. Biotechnology and bioengineering 100(6):1205-1213.

29. Uttamsingh, S., X. Bao, K. T. Nguyen, M. Bhanot, J. Gong, J. L. K. Chan, F. Liu, T. T. Chu, and L. H. Wang. 2008. Synergistic effect between EGF and TGF- $\beta 1$ in inducing oncogenic properties of intestinal epithelial cells. Oncogene 27(18):2626-2634.

30. Pang, M., A. Georgoudaki, L. Lambut, J. Johansson, V. Tabor, K. Hagikura, Y. Jin, M. Jansson, J. Alexander, and C. M. Nelson. 2016. TGF- $\beta 1$-induced EMT promotes targeted migration of breast cancer cells through the lymphatic system by the activation of CCR7/CCL21-mediated chemotaxis. Oncogene 35(6):748-760.

31. Gore, J., I. E. Imasuen-Williams, A. M. Conteh, K. E. Craven, M. Cheng, and M. Korc. 2016. Combined targeting of TGF- $\beta$, EGFR and HER2 suppresses lymphangiogenesis and metastasis in a pancreatic cancer model. Cancer Letters 379(1):143-153.

32. Sempere, L. F., J. R. Gunn, and M. Korc. 2011. A novel 3-dimensional culture system uncovers growth stimulatory actions by TGF $\beta$ in pancreatic cancer cells. Cancer Biol. Ther. 12(3):198-207.

33. Whipple, C. A., A. L. Young, and M. Korc. 2011. A KrasG12D-driven genetic mouse model of pancreatic cancer requires glypican-1 for efficient proliferation and angiogenesis. Oncogene 31:2535. Original Article. 
34. Varennes, J., H.-r. Moon, S. Saha, A. Mugler, and B. Han. 2019. Physical constraints on accuracy and persistence during breast cancer cell chemotaxis. PLOS Computational Biology 15(4):e1006961.

35. Harley, B. A., H.-D. Kim, M. H. Zaman, I. V. Yannas, D. A. Lauffenburger, and L. J. Gibson. 2008. Microarchitecture of three-dimensional scaffolds influences cell migration behavior via junction interactions. Biophysical journal 95(8):4013-4024.

36. Chavez, K. J., S. V. Garimella, and S. Lipkowitz. 2010. Triple negative breast cancer cell lines: One tool in the search for better treatment of triple negative breast cancer. Breast Dis 32:35-48.

37. Wang, S.-J., W. Saadi, F. Lin, C. Minh-Canh Nguyen, and N. Li Jeon. 2004. Differential effects of EGF gradient profiles on MDA-MB-231 breast cancer cell chemotaxis. Experimental Cell Research 300(1):180-189.

38. Seeley, E. S., C. Carrière, T. Goetze, D. S. Longnecker, and M. Korc. 2009. Pancreatic cancer and precursor pancreatic intraepithelial neoplasia lesions are devoid of primary cilia. Cancer research 69(2):422-430.

39. Bradney, M. J., S. M. Venis, Y. Yang, S. F. Konieczny, and B. Han. 2020. A Biomimetic Tumor Model of Heterogeneous Invasion in Pancreatic Ductal Adenocarcinoma. Small:1905500.

40. Levchenko, A., and P. A. Iglesias. 2002. Models of eukaryotic gradient sensing: application to chemotaxis of amoebae and neutrophils. Biophysical journal 82(1):50-63.

41. Ellison, D., A. Mugler, M. D. Brennan, S. H. Lee, R. J. Huebner, E. R. Shamir, L. A. Woo, J. Kim, P. Amar, and I. Nemenman. 2016. Cell-cell communication enhances the capacity of cell ensembles to sense shallow gradients during morphogenesis. Proceedings of the National Academy of Sciences 113(6):E679-E688.

42. Varennes, J., S. Fancher, B. Han, and A. Mugler. 2017. Emergent versus individual-based multicellular chemotaxis. Physical review letters 119(18):188101.

43. Muller, P. A., K. H. Vousden, and J. C. Norman. 2011. p53 and its mutants in tumor cell migration and invasion. Journal of Cell Biology 192(2):209-218.

44. Moustakas, A., and C.-H. Heldin. 2008. Dynamic control of TGF- $\beta$ signaling and its links to the cytoskeleton. FEBS Letters 582(14):2051-2065.

45. Hou, Y., S. Hedberg, and I. C. Schneider. 2012. Differences in adhesion and protrusion properties correlate with differences in migration speed under EGF stimulation. BMC biophysics 5(1):8.

46. Endres, R. G., and N. S. Wingreen. 2008. Accuracy of direct gradient sensing by single cells. Proceedings of the National Academy of Sciences 105(41):15749-15754.

47. Clark, A. G., and D. M. Vignjevic. 2015. Modes of cancer cell invasion and the role of the microenvironment. Current opinion in cell biology 36:13-22.

48. Gandalovičová, A., T. Vomastek, D. Rosel, and J. Brábek. 2016. Cell polarity signaling in the plasticity of cancer cell invasiveness. Oncotarget 7(18):25022-25049.

49. Stuelten, C. H., C. A. Parent, and D. J. Montell. 2018. Cell motility in cancer invasion and metastasis: insights from simple model organisms. Nature Reviews Cancer 18(5):296-312.

50. Corallino, S., C. Malinverno, B. Neumann, C. Tischer, A. Palamidessi, E. Frittoli, M. Panagiotakopoulou, A. Disanza, G. Malet-Engra, and P. Nastaly. 2018. A RAB35p85/PI3K axis controls oscillatory apical protrusions required for efficient chemotactic migration. Nature communications 9(1):1-19. 


\section{Acknowledgments}

This work was partially supported by a grant from the Purdue University Center for Cancer

Research, NIH grant P30 CA023168.

\section{Author contributions}

$\mathrm{AM}$ and $\mathrm{BH}$ conceived the idea. HM and SS performed the research and acquired the data. All authors discussed the results and wrote the manuscript.

\section{Competing interests}

The authors declare that they have no competing interests.

\section{Supporting Materials}

Chemotaxis Platform

Mathematical Model

Fig. S1. Chip design and chemotaxis characteristics.

Fig. S2. Cell trajectories of all collected trajectories.

Fig. S3. Effect of $\mathrm{M}_{0}$ on $\mathrm{CI}$.

Fig. S4. Effect of dimensionality on CI.

Fig. S5. CI distribution of all collected trajectories.

Fig. S6. Speed distribution of all collected trajectories.

Movie S1. Time-lapse of cell migration. 


\section{Supporting Materials}

\section{Chemotaxis Platform}

To investigate the cellular response to the gradient signals by observing the cell migratory behaviors, we use an engineered chemotaxis platform developed in a microfluidic device, as shown in Fig. S1. The chemotaxis platform was designed for engineering the chemical environment surrounding the cells embedded in the 3D extracellular matrix. The platform is composed of three microfluidic channels with $100 \mu \mathrm{m}$ in thickness. A center channel of $1 \mathrm{~mm}$ wide, which aims to contain cells with a collagen matrix, is located in between source (top) and sink (bottom) channels with $300 \mu \mathrm{m}$ wide. At the end ports, the source and sink channels are connected to large reservoirs so that the culture medium can be supplemented through the channels. For the cell culture, the basic culture medium is filled in both source and sink channels. In order to develop a concentration gradient in the center channel, growth factor solution (TGF- $\beta$ or EGF) based on the medium is added through the source channel. On the other hand, the sink channel is filled with growth factorfree medium. Assuming that there is neither flow nor any pressure differences between the channels, the concentration of the given growth factor $(i)$ can be illustrated by the conservation equation of chemical species:

$$
\frac{\partial C_{i}}{\partial t}=D_{i} \cdot \nabla C_{i}
$$

At the steady state, the concentration profile in the center channel goes to a linear. The linear profile can persist for a while with an assumption that is the concentration at the source and side channels are constant with a large volume of reservoirs. Consequently, cells cultured in the collagen matrix are exposed to a linear gradient of a specific soluble factor. The detailed technique 
to develop the microfluidic device and the diffusion principle in the platform was described in our previous study (1).

\section{Mathematical Model}

In our model there are finite number of $\mathrm{M}$ molecules residing inside the surface of a spherical cell.

There is a continuous field of TGF- $\beta$ (T) and EGF (E) molecules outside the cell with linear concentration profiles as in the experiments. When a $\mathrm{T}$ or $\mathrm{E}$ molecule comes in contact with the cell surface it converts an $\mathrm{M}$ molecule at the same location inside the cell to $\mathrm{M}^{*}$ according to the reactions in Eq. 1. The dynamics of the migration molecule in each half of the cell denoted by $i(i$ $=1,2$ for left/right half of the cell) is given by,

$$
\frac{d M_{i}^{*}}{d t}=k_{T} T_{i} M_{i}+k_{E} E_{i} M_{i}-M_{i}^{*}
$$

The steady state value of the migration molecule is,

$$
M_{i}^{*}=k_{T} T_{i} M_{i}+k_{E} E_{i} M_{i}
$$

The signal due to the migration molecule is the difference of the migration molecule number between the two halves and is given by,

$$
\overline{M_{2}^{*}-M_{1}^{*}}=\overline{\Delta M^{*}}=k_{T}\left(\overline{M_{2} \Delta T+T_{1} \Delta M}\right)+k_{E}\left(\overline{M_{2} \Delta E+E_{1} \Delta M}\right)
$$

We assume that the mean number of molecules in two halves is much greater than the difference since the relative gradient of TGF- $\beta$ or EGF is very small $\left(\operatorname{Rg}_{\mathrm{T}(\mathrm{E})} \ll \mathrm{T}_{0}\left(\mathrm{E}_{0}\right)\right)$. Using this assumption, we can say, 


$$
\overline{M_{2}}=\overline{M_{1}}=\frac{\bar{M}}{2}
$$

and so on for all the relevant fields of TGF- $\beta$ and EGF. We use this approximate value of the mean number of molecules in each half to rewrite the migration molecule signal as,

$$
\overline{\Delta M^{*}}=\frac{k_{T}}{2}(\overline{M \Delta T+T \Delta M})+\frac{k_{E}}{2}(\overline{M \Delta E+E \Delta M})
$$

Now since the total number of $\mathrm{M}$ or $\mathrm{M}^{*}$ molecule is constant $(\mathrm{M} 0)$ we can use the following result to derive the signal.

$$
\overline{\Delta M}=-\overline{\Delta M^{*}}
$$

And the signal due to the migration molecule is given by,

$$
\overline{\Delta M^{*}}=\frac{\bar{M}\left(\frac{k_{T} \overline{\Delta T}+k_{E} \overline{\Delta E}}{2}\right)}{\left(1+\frac{k_{T} \bar{T}+k_{E} \bar{E}}{2}\right)}
$$

We can derive the mean number of $\mathrm{M}$ molecules as,

$$
\bar{M}+\overline{M^{*}}=M_{0}=\bar{M}\left(1+k_{T} \bar{T}+k_{E} \bar{E}\right) \rightarrow \bar{M}=\frac{M_{0}}{1+k_{T} \bar{T}+k_{E} \bar{E}}
$$

We can use,

$$
\overline{\Delta T(E)}=R V g_{T(E)}=\frac{a V g_{T(E)}}{2} ; \overline{T(E)}=V T_{0}\left(E_{0}\right)
$$


to derive the signal for the migration molecule $\mathrm{M}^{*}$ which is given by,

$$
\overline{\Delta M^{*}}=\frac{\frac{M_{0} V a}{2}\left(\frac{k_{T} g_{T}+k_{E} g_{E}}{2}\right)}{\left(1+\frac{k_{T} V T_{0}+k_{E} V E_{0}}{2}\right)\left(1+k_{T} V T_{0}+k_{E} V E_{0}\right)}
$$

Where $a, R$ and $V$ is the size, radius and volume of the cell respectively. In order to derive the variance in the signal of the migration molecule we define the event "success" as the conversion of $\mathrm{M}$ to $\mathrm{M}^{*}$ molecule. The probability of success in each half $i=1,2$ is,

$$
P_{i}=\frac{k_{T} \bar{T}_{\imath}+k_{E} \bar{E}_{\imath}}{1+k_{T} \bar{T}_{\imath}+k_{E} \bar{E}_{\iota}}=\frac{V\left(k_{T} T_{0}+k_{E} E_{0}\right) / 2}{1+V\left(k_{T} T_{0}+k_{E} E_{0}\right) / 2}
$$

Because the reaction is a binomial process with the probability of converting $M$ to $M^{*}$ given by $P_{i}$ and the mean number of molecules in both halves roughly the same, the noise in $\Delta \mathrm{M}^{*}$ is,

$$
\sigma_{\Delta M^{*}}^{2}=\sigma_{M_{1}^{*}}^{2}+\sigma_{M_{2}^{*}}^{2}=\frac{M_{0}}{2}\left(P_{1}\left(1-P_{1}\right)+P_{2}\left(1-P_{2}\right)\right)=\frac{\frac{M_{0} V\left(k_{T} T_{0}+k_{E} E_{0}\right)}{2}}{\left(1+\frac{V\left(k_{T} T_{0}+k_{E} E_{0}\right)}{2}\right)^{2}}
$$

Thus, the signal to noise ratio (SNR) in presence of both TGF- $\beta$ and EGF gradient is given by,

$$
S N R^{2}=\frac{{\overline{\Delta M^{*}}}^{2}}{\sigma_{\Delta M^{*}}^{2}}=\frac{\frac{M_{0} V^{2} a^{2}}{2}\left(\frac{k_{T} g_{T}+k_{E} g_{E}}{2}\right)^{2}}{\left(1+k_{T} V T_{0}+k_{E} V E_{0}\right)^{2}\left(k_{T} V T_{0}+k_{E} V E_{0}\right)}
$$


Our mathematical calculation tells us that the SNR should increase with $\mathrm{M}_{0}$ as $\sqrt{ } \mathrm{M}_{0}$. We know this is an approximation since although SNR can take any values, but CI has an upper bound of 1 . So, it is interesting to see how does $\mathrm{CI}$ changes with $\mathrm{M}_{0}$ in the simulations. On simulating the migration against TGF- $\beta$ gradient for different values of $\mathrm{M}_{0}$ we see that the median (Fig. S4) of CI increases with increase of $\mathrm{M}_{0}$ but gradually saturates to 1 . We choose the particular value of $\mathrm{M}_{0}$ for our other simulation so that the CI value matches with that in the experiment $\left(\mathrm{M}_{0}=5000\right)$.

\section{Effect of dimensionality of space on CI}

We use a circular cell doing a 2-D biased random walk to determine the CI in simulations. In reality the cell is a 3-D object moving in 3-D space with the gradient in one direction. To understand the effect of dimensionality on the CI calculation we do the following simulations:

1. A 2-D biased random walk by a cell where after moving with a constant speed $\mathrm{s}$ for time $\tau$ a new direction $\varphi$ is chosen from the distribution,

$$
P_{\phi}(\phi)=\frac{1+\epsilon \cos (\phi)}{2 \pi} ; \phi: 0-2 \pi
$$

$\varepsilon$ being the bias parameter.

2. A 3-D biased random walk by a cell where after moving with the same constant speed s for time $\tau$ a new direction $(\varphi, \theta)$ is chosen from the distribution,

$$
P_{\phi}(\phi)=\frac{1+\epsilon \cos (\phi)}{2 \pi}, P_{\theta}(\theta)=\frac{1}{2} ; \phi: 0-2 \pi, \theta: 0-\pi
$$


From the simulations for different values of $\varepsilon$ we infer that the median (Fig. S5) of CI for both 2D and 3D are both quantitatively and qualitatively similar to each other for different values of $\varepsilon$. This result justifies performing simulations with a circular cell in 2-D.

\section{Condition for antagonism}

Using our mathematical model, we can show,

$$
S N R_{E}=\frac{\eta+\beta}{(1+\beta) \sqrt{\eta}} S N R_{T}
$$

where, $\operatorname{SNRE}(\mathrm{T})$ is the signal to noise ratio (SNR) in presence of only $\mathrm{EGF}(\mathrm{TGF}-\beta)$ gradient.

When both gradients are present the SNR is given by,

$$
S N R_{T, E}=\frac{(\eta+\beta) \sqrt{1+\eta}}{(1+\eta+\beta) \sqrt{\eta}} S N R_{T}
$$

To understand the condition for antagonism we need satisfy both the conditions $\mathrm{SNR}_{\mathrm{T}, \mathrm{E}}<\mathrm{SNR}_{\mathrm{T}}$ and $\mathrm{SNR}_{\mathrm{T}, \mathrm{E}}<\mathrm{SNR}_{\mathrm{E}}$. For $\mathrm{SNR}_{\mathrm{T}, \mathrm{E}}<\mathrm{SNR}_{\mathrm{T}}$ the equation for the line is,

$$
1>\frac{(\eta+\beta) \sqrt{1+\eta}}{(1+\eta+\beta) \sqrt{\eta}} \rightarrow \beta<\sqrt{\eta(1+\eta)}
$$

And $\mathrm{SNR}_{\mathrm{T}, \mathrm{E}}<\mathrm{SNR}_{\mathrm{E}}$ gives us the line,

$$
\frac{(\eta+\beta) \sqrt{1+\eta}}{(1+\eta+\beta) \sqrt{\eta}}<\frac{\eta+\beta}{(1+\beta) \sqrt{\eta}} \rightarrow \beta<\sqrt{1+\eta}
$$

So, for antagonism condition we need, 
bioRxiv preprint doi: https://doi.org/10.1101/2020.12.10.419572; this version posted December 11,2020 . The copyright holder for this preprint (which was not certified by peer review) is the author/funder, who has granted bioRxiv a license to display the preprint in perpetuity. It is made available under aCC-BY-NC-ND 4.0 International license.

$$
\beta<\sqrt{\eta(1+\eta)} \& \beta<\sqrt{1+\eta} \quad \forall \eta
$$

Which is equivalent to,

$$
\beta<\left\{\begin{array}{rr}
\sqrt{\eta(1+\eta)}, & \eta<1 \\
\sqrt{1+\eta}, & \eta \geq 1
\end{array}\right.
$$




\section{A PDMS}

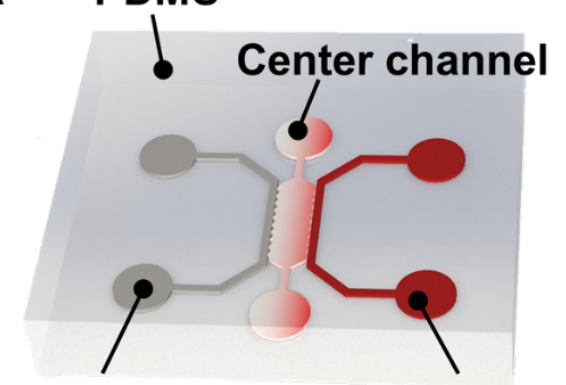

Sink channel Source channel
B

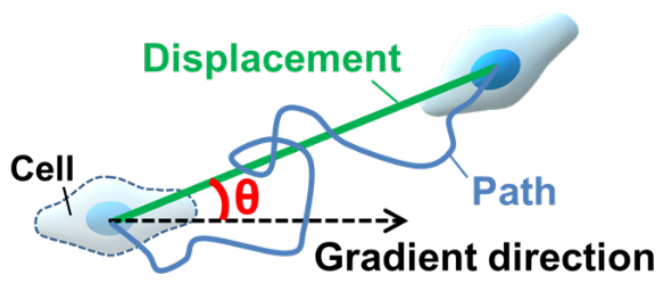

Fig. S1. Chip design. (A) Schematic description of a microfluidic chemotaxis platform to induce the chemical gradient. (B) Characterization of the measured cell trajectory
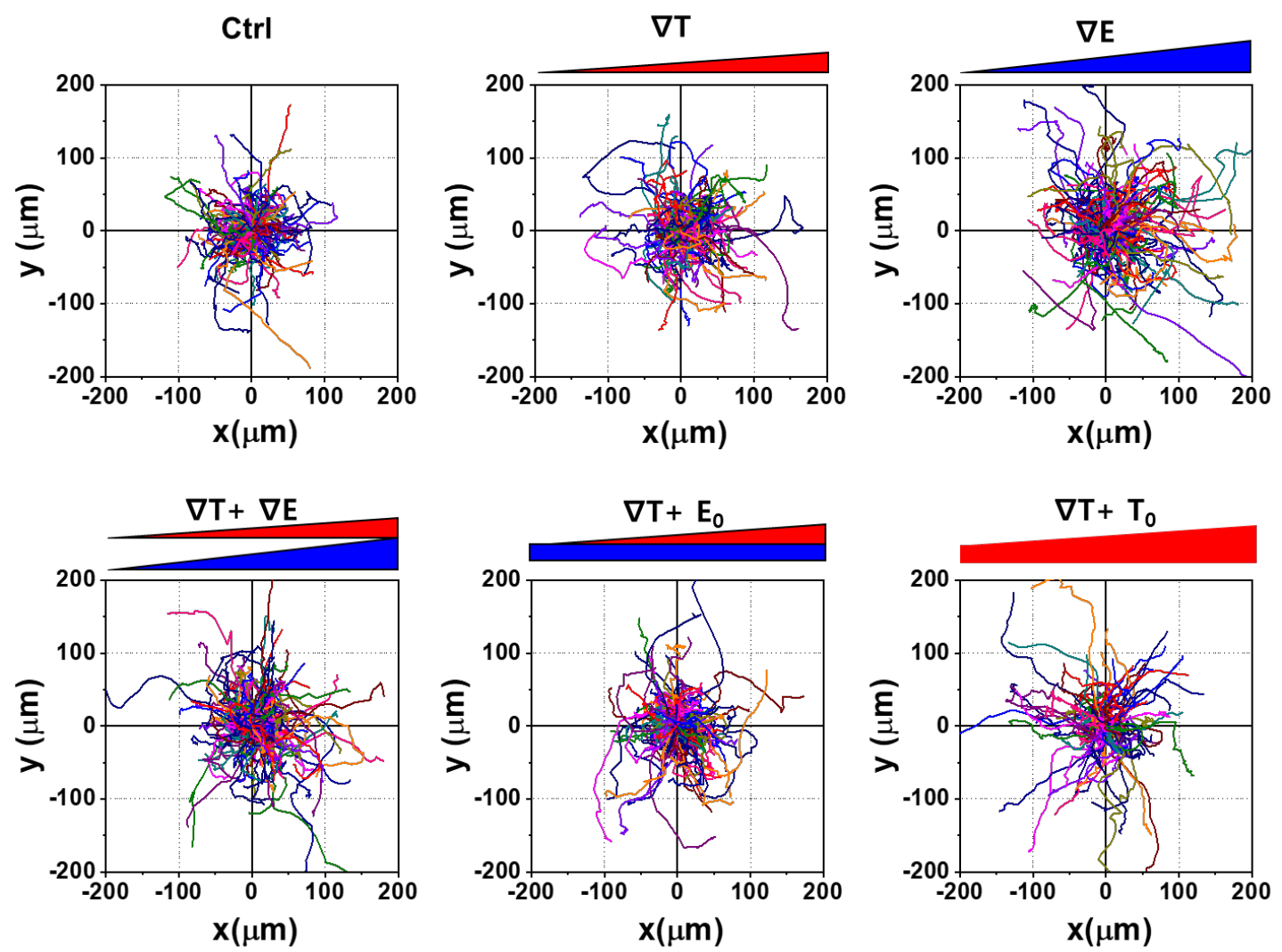

Fig. S2. All collected trajectories of MDA-MB-231. Control, $50 \mathrm{nM} / \mathrm{mm}$ TGF- $\beta$ gradient (VT), $800 \mathrm{nM} / \mathrm{mm}$ EGF gradient ( $\nabla \mathrm{E})$, combined gradients of $50 \mathrm{nM} / \mathrm{mm}$ TGF $-\beta$ with $800 \mathrm{nM} / \mathrm{mm}$ EGF $(\nabla T+\nabla E)$, combination of $50 \mathrm{nM} / \mathrm{mm}$ TGF- $\beta$ gradient with $400 \mathrm{nM} E G F$ background $\left(\nabla T+E_{0}\right)$, and combination of $50 \mathrm{nM} / \mathrm{mm}$ TGF- $\beta$ gradient with $200 \mathrm{nM}$ TGF- $\beta$ background $\left(\nabla T+\mathrm{T}_{0}\right)$. Trajectories are collected from 116-233 cells in a condition. 


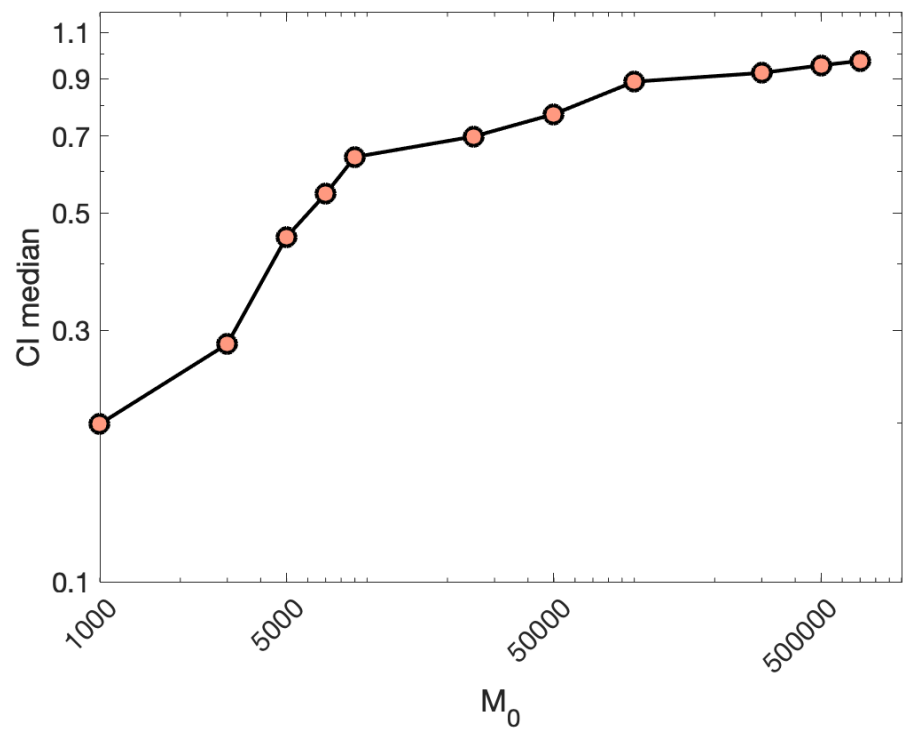

Fig. S3. Effect of $\mathrm{M}_{0}$ on $\mathrm{Cl}$. Median of $\mathrm{Cl}$ increases with increase in the number of total available migration molecules Mo.

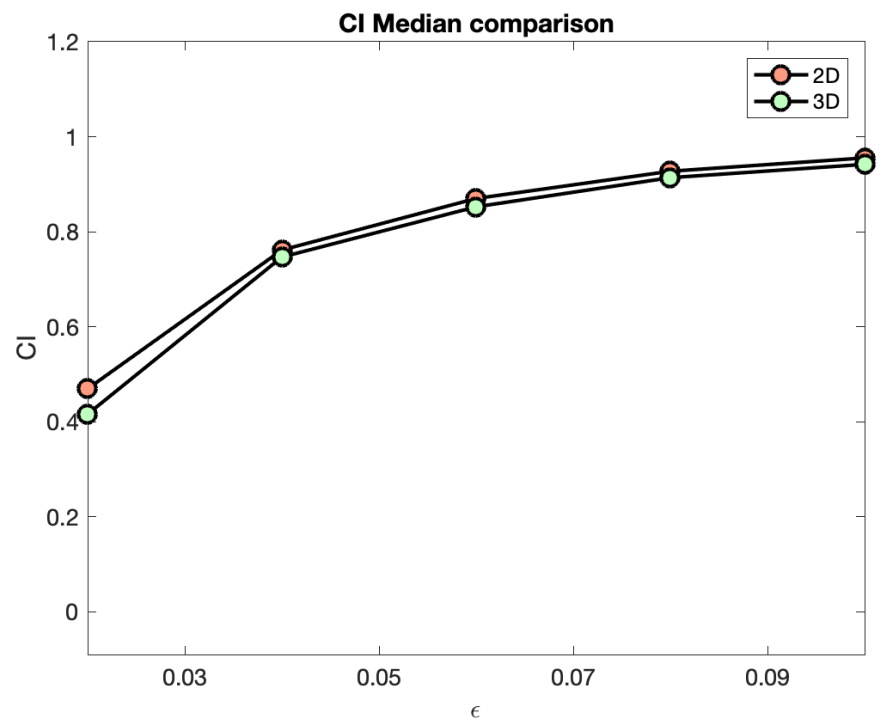

Fig. S4. Effect of dimensionality on $\mathrm{Cl}$. Median of $\mathrm{Cl}$ is quantitatively and qualitatively similar for 2-D and 3-D case and increases with the increase in the bias $\varepsilon$. 

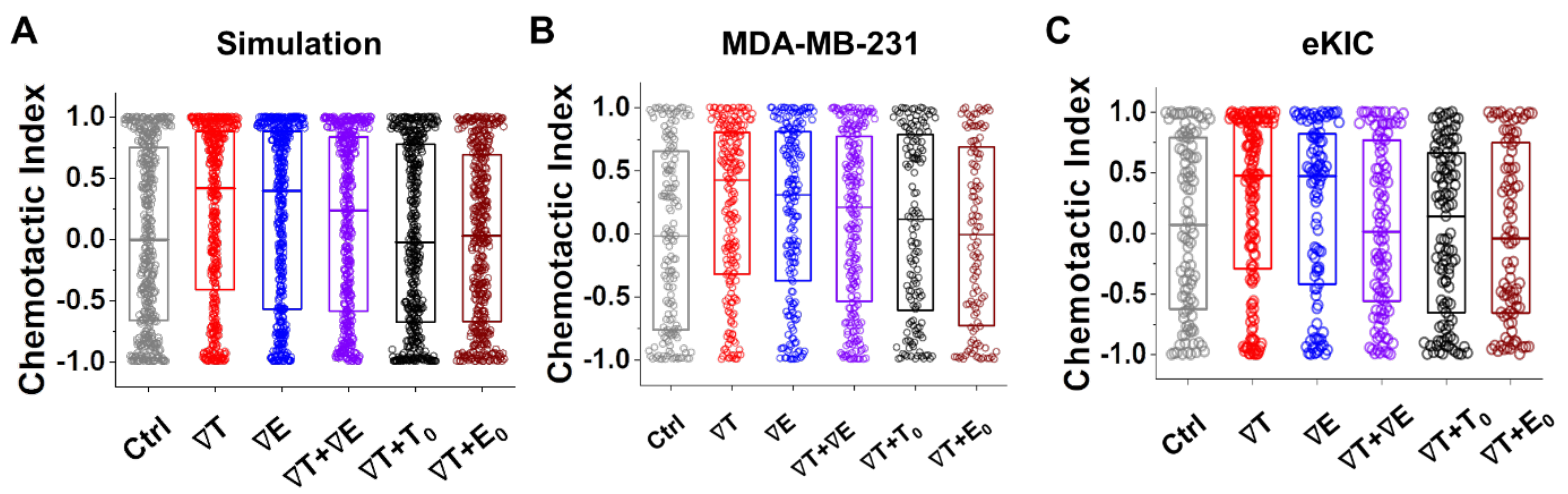

Fig. S5. $\mathrm{Cl}$ distributions of collected trajectories from all groups. (A) simulation (400 trajectories /condition). (B) MDA-MB-231 and (C) eKIC ; Bar: medians \pm S.E Dot: Cl from a single trajectory. $(B, C)>100$ trajectories/condition. Box: quartiles with a median line in the middle of the box.
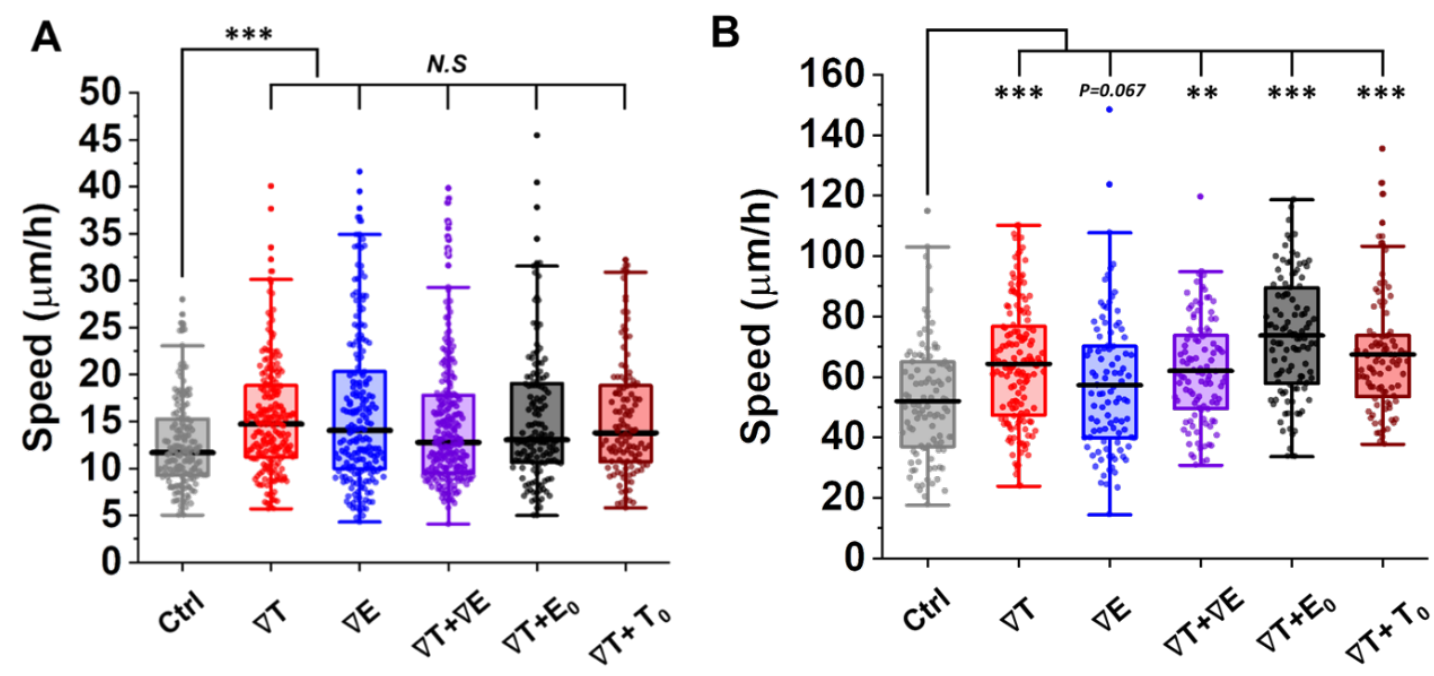

Fig. S6. Speed distribution of collected trajectories from all experimental groups. (A) MDAMB-231 - Control (ctrl), $50 \mathrm{nM} / \mathrm{mm}$ TGF- $\beta$ gradient $(\nabla \mathrm{T}), 800 \mathrm{nM} / \mathrm{mm}$ EGF gradient ( $\nabla \mathrm{E})$, combined gradients of $50 \mathrm{nM} / \mathrm{mm}$ TGF $-\beta$ with $800 \mathrm{nM} / \mathrm{mm}$ EGF $(\nabla T+\nabla E)$, combination of $50 \mathrm{nM} / \mathrm{mm}$ TGF- $\beta$ gradient with $400 \mathrm{nM}$ EGF background $\left(\nabla T+\mathrm{E}_{0}\right)$, and combination of $50 \mathrm{nM} / \mathrm{mm}$ TGF- $\beta$ gradient with 200nM TGF- $\beta$ background $\left(\nabla T+T_{0}\right)$ and (B) eKIC - ctrl, $10 \mathrm{nM} / \mathrm{mm} \nabla T, 200 \mathrm{nM} / \mathrm{mm}$ EGF gradient $(\nabla E)$, combined $10 \mathrm{nM} / \mathrm{mm} \nabla T+200 \mathrm{nM} / \mathrm{mm} \nabla \mathrm{E}(\nabla \mathrm{T}+\nabla \mathrm{E})$, combination of $10 \mathrm{nM} / \mathrm{mm} \nabla T$ with $100 \mathrm{nM}$ EGF background $\left(\nabla \mathrm{T}+\mathrm{E}_{0}\right)$, and combination of $10 \mathrm{nM} / \mathrm{mm} \nabla \mathrm{T}$ with $50 \mathrm{nM}$ TGF- $\beta$ background $\left(\nabla T+T_{0}\right)$; Dot: speed from a single trajectory. Box: interquartile range (IQR) \pm 1.5 IQR whiskers with a mean line in the middle of the box. ${ }^{*}: p<.05,{ }^{* * *}: p<.001$ (ANOVA Tukey's post hoc test). 
bioRxiv preprint doi: https://doi.org/10.1101/2020.12.10.419572; this version posted December 11,2020 . The copyright holder for this preprint

(which was not certified by peer review) is the author/funder, who has granted bioRxiv a license to display the preprint in perpetuity. It is made available under aCC-BY-NC-ND 4.0 International license.

\section{Movie S.1}

\section{Supporting References}

1. Varennes, J., H.-r. Moon, S. Saha, A. Mugler, and B. Han. 2019. Physical constraints on accuracy and persistence during breast cancer cell chemotaxis. PLOS Computational Biology 15(4):e1006961. 